\title{
Atomic Mass Knowledge: 1999 Status
}

\author{
G. Audi a and A.H. Wapstra ${ }^{\mathrm{b}}$ \\ ${ }^{a}$ Centre de Spectrométrie Nucléaire et de Spectrométrie de Masse,CSNSM, IN2P3-CNRS, \\ Bâtiment 108, F-91405 Orsay Campus, France \\ b National Institute of Nuclear Physics and High-Energy Physics, NIKHEF, PO Box 41882, \\ 1009DB Amsterdam, The Netherlands
}

\begin{abstract}
An overview is given of developments of interest for the determination of atomic masses of atoms around nuclear ground-states.
\end{abstract}

\section{About the back-bone}

In this report we discuss data new since our 1995 "update" (1) (which we will denote here Ame95) of our 1993 mass table (2). For several cases, we will mention work in progress, of which we assume that pertinent results will be available before we finish next mass evaluation. For this reason our title refers not to today but to the near future.

Not only for this Conference but also for nuclear physics in general, the most important new facts are concerned with nuclides far removed from the line of stability against $\beta$ decay. Yet, some new data for nuclides along that line deserve notice. We will therefore devote some attention to them. This will also serve to show several difficulties of the type we have to deal with in our evaluations.

We know people are working on the greatest problem along the line of $\beta$-stability: the masses of the stable $\mathrm{Hg}$ isotopes. For them, mass spectroscopic results from 1980 (3) deviate many $\mathrm{keV}$, far outside the reported errors, from the results derived from reaction chains combined with spectroscopic results for other elements. Preliminary new mass spectroscopic results on one $\mathrm{Hg}$ isotope seem to agree with the earlier one. Though the precision is still rather less than that reported for the earlier result, a great problem would arise if it would be confirmed.

New results start to emerge about the most fundamental masses. The Stockholm Smile group (4), working with a Penning trap, starts to get results of which they were so kind to send us provisional values. Their new value for $\mathrm{H}$ may be even somewhat more precise than the ones used in AME95 and agrees perfectly with them. But the new mass value for ${ }^{4}$ He may deviate somewhat.

There are also developments that will affect the mass value for the neutron. It is derived essentially by combining the mass spectroscopic results for $\mathrm{H}$ and $\mathrm{D}$ with the energy of the $\gamma$-rays emitted in the capture of thermal neutrons in hydrogen. The most precise value for the latter is derived from measurements of its wavelength, by diffraction in a silicon crystal (5). Unfortunately, there was some uncertainty about the value of its lattice constant. It is hoped, that the current evaluation by Taylor and Cohen of data, important for determination of fundamental physical constants, will improve the situation. Also, it has been reported to us that a new measurement is in progress.

Somewhat less accurate $\gamma$-energy values are obtained by measurements in semiconductor diodes. These are calibrated, essentially, with the $411 \mathrm{keV} \gamma$-ray of ${ }^{198} \mathrm{Au}$, which is again measured by the Deslattes group (6). They may also be revised somewhat. 
Van der Leun and Helmer are working on the general calibration of $\gamma$-rays. We are, in line with this, reconsidering the calibrations for many $(n, \gamma)$ reactions, and so find that several old neutron binding energies can be improved. Following case presents an illustration. A value with a somewhat large error $(650 \mathrm{eV})$ was reported (7) for the neutron binding energy in ${ }^{54} \mathrm{Cr}$. Studying the paper taught, that this value was essentially the sum of the energies of two capture $\gamma$-rays of nearly the same enery. In recent work, a much improved value was given for one of them. And since the original paper gave a rather precise value for the difference in energy of the two rays, we can derive a much improved value for the resulting neutron binding energy.

The calibration for precision particle energies, e.g. for $(p, \gamma)$ reactions and (p,n) thresholds we treat too. Unfortunately, new data (8) reportedly more precise (about $20 \mathrm{ppm}$ ) than old ones differ rather more than expected (more than $100 \mathrm{ppm}$ ) from older ones, causing yet unsolved difficulties.

The SMILE group also measured mass values for ${ }^{22} \mathrm{Ne},{ }^{36} \mathrm{Ar}$ and ${ }^{133} \mathrm{Cs}$. The provisional results give rise to the following comments.

The ${ }^{36} \mathrm{Ar}$ result is some $1.2 \mathrm{keV}$ lower than the AME95 value, to which an error of $0.3 \mathrm{keV}$ was assigned. The latter value is, essentially, due to mass spectroscopic results for ${ }^{35} \mathrm{Cl}$ and ${ }^{37} \mathrm{Cl}$, combined with reaction energies for 5 reactions. These data do agree quite well if combined in a least squares analysis: $R_{e} / R_{i}=1.13$. But if the (provisional) new value for ${ }^{36} \mathrm{Ar}$ is added, $R_{e} / R_{i}$ is increased to 2.00 . But this value is reduced to a reasonable 1.35 if, of the two available values for the ${ }^{36} \mathrm{Ar}(\mathrm{n}, \gamma)^{37} \mathrm{Ar}$ reaction energy, the oldest not well documented one is no longer used.

The ${ }^{22} \mathrm{Ne}$ result agrees quite well with the earlier ${ }^{20} \mathrm{Ne}$ value combined with the neutron capture ray energies in ${ }^{20} \mathrm{Ne}$ and ${ }^{21} \mathrm{Ne}$ mentioned in AME95 - but is over ten times more precise. Yet, the mass situation in this region is not perfect either. The mass of ${ }^{28} \mathrm{Si}$ is known with high precision. Its difference with that of ${ }^{22} \mathrm{Ne}$ also follows from a bridge of some 4 reaction energies. The so derived value was already in a not perfect agreement with the mass spectroscopic data in Ame95. The new ${ }^{22} \mathrm{Ne}$ result makes it worse: the difference is over $1 \mathrm{keV}$, rather far outside the reported errors. This situation too requires a further analysis.

The SMILE ${ }^{133}$ Cs result is important for the determination of masses many Cs and Ba isotopes: as discussed below, their relations with ${ }^{133} \mathrm{Cs}$ have been determined mass spectroscopically. The (provisional) SMILE value is about $5 \mathrm{keV}$ higher than the AmE95 one, to which an error of $3 \mathrm{keV}$ had been assigned. The latter is mainly the result of a set of connections, through known Cs $\beta^{+}$decay energies, with Xe nuclides, for which mass spectroscopic mass values were available (see the scheme fig. 1 in (2).) The nearest ones are those at mass numbers 124, 128, 129, 130 and 132. Analyzing them, we find that the connection with ${ }^{132} \mathrm{Xe}$ would make ${ }^{133} \mathrm{Cs} 15(7) \mathrm{keV}$ higher, that with ${ }^{124} \mathrm{Xe} 35(20)$ lower. The first one, thus, is improved by the SMILE result. The other connections are not severely affected. In total, specifically, this analysis throws some doubt on the ${ }^{125} \mathrm{Cs}$ $\beta^{+}$decay energy.

\section{The NUBASE evaluation}

Already since long, we maintain a file of approximate mass values as input in our computer programs. (Mfile. Essentially, these programs calculate the differences with the input values.) In cases where isomers occur, one has to be careful to check which one 
is involved in reported experimental data, such as $\beta$-decay energies. Cases have occured where authors were not (yet) aware of isomeric complications. For that reason, our Mfile contained known data on such isomeric pairs (half-lives; excitation energies).

The matter of isomerism became even more important, when mass spectroscopic methods were developed to measure masses of exotic atoms, far from $\beta$-stability and therefore having small half-lives. The resolution was then limited, often insufficient to separate isomers. Then, one so obtains an average mass of the isomeric pair. A mass of the ground-state, our primary purpose, can then only be derived if one has information on the excitation energy and on the production rates of the two isomers. And in cases where e.g. the excitation energy was not known, it might be estimated by extrapolation of the values for isomers or isotones in the neighbourhood. We therefore decided, that it might be useful to make our Mfile as complete as possible. This turned out to be a major job. And since it was judged possible, that the result might be useful for others, it was published (9).

\section{Mass spectroscopic exotic results}

A group, originally from Mainz but working at ISOLDE in Geneva, developed a method of measuring masses of radio-active isotopes in a Penning trap. Mass values of many isotopes of Cs and some of Ba were already used in our 1993 mass evaluation, and will soon be published fully (10). These new masses were derived from comparisons with the mass of ${ }^{133} \mathrm{Cs}$ and therefore may change somewhat in a new evaluation, as discussed above.

With this IsOLTRAP, masses (11) of several neutron-poor isotopes of lighter rare earth isotopes have also been determined, with a precision of about $20 \mathrm{keV}$. Especially mentioned may be their results for ${ }^{148} \mathrm{Dy},{ }^{149} \mathrm{Dy}$ and ${ }^{150} \mathrm{Ho}$. These nuclides are the endpoints of $\alpha$-decay chains, for which $\alpha$-particle energies are known, starting with ${ }^{180} \mathrm{~Pb},{ }^{181} \mathrm{~Pb}$ and ${ }^{174} \mathrm{Au}$ respectively. No earlier value was known for ${ }^{150} \mathrm{Ho}$; the value for ${ }^{148} \mathrm{Dy}$ is a decided improvement. Reversely, they found a value for ${ }^{158}$ Dy which agreed quite well with the earlier value derived from its two member $\alpha$-decay chain.

Finally, in a private communication we learned of their measurements on $\mathrm{Hg}$ isotopes with mass numbers from 184 to 200, with a precision of the order of $20 \mathrm{keV}$. They were obtained by comparison with ${ }^{208} \mathrm{~Pb}$; and thus are affected with the uncertainty about the mass of the stable $\mathrm{Hg}$ isotopes mentioned above. Especially usefull was, that in several cases they could separate isomers, at the odd mass numbers. Thus, in combination with $\alpha$-decay data, good information is obtained for even- $Z$ nuclei between ${ }^{176} \mathrm{Pt}$ and ${ }^{210} \mathrm{Th}$. These data, combined with $\operatorname{Pb}(\alpha)$ energies, allow a check on neutron pairing energies in proton-rich $\mathrm{Hg}$ and $\mathrm{Pb}$ isotopes. The Jensen-Hansen-Jonson (12) estimate is decidedly better than the earlier formula $(12 \mathrm{MeV}) / A^{1 / 2}$.

A quite exciting development occured at Gsi, Darmstadt. They succeeded in storing radio-active ions in a storage ring and determining their masses (13) by, essentially, measuring their cyclotron frequencies. Those atoms were produced by bombarding targets with heavy ions, and caught in flight. As mentioned in two Muenich theses (14),(15), masses were so determined for many dozens of proton-rich nuclides, roughly in the mass region $A=140-200$. Between many of the measured nuclides, connections exist due to chains of $\alpha$-decays. Thus, checks are possible; and masses can be derived for many other nuclides. 


\section{Measurements of proton decays}

Our Ame95 used a few results of measurements of energies of protons emitted in proton decay, made in Daresbury. Now we posses new measurements, first from the same place (16),(17), but later at Argonne National Laboratory (18),(19) and Oak Ridge National Laboratory (20) in collaboration with groups there. Thus, data were obtained for several very proton-rich nuclides, from ${ }^{131} \mathrm{Eu}$ to ${ }^{195} \mathrm{Bi}$.

These data are quite important, mainly for two reasons. In the first place, we apply systematics of some quantities (among them proton separation energies) for estimating mass values for nuclides, for which no experimental mass data are available. For this purpose, knowledge of proton separation energies just beyond the proton drip line is quite valuable.

In the second place, the properties of proton decay allow in several cases to find proton decay energies from both members of an isomeric pair. Since, often, both are observed to decay to the ground-state of the daughter, one so derives the excitation energy of the isomer. And these studies even allow to get a fair estimate of the spin-parities of the separate members.

The advantage of this is especially valuable, if for both members $\alpha$-decay is observed. In a particular case, even a succession of several such decays was found. Their study showed that decays for some of these daughters observed earlier work did not belong to ground-states, as assumed earlier; with evident consequences for the masses assigned to them.

\section{The $\alpha$-decay chains}

Measured $\alpha$-decay energies in such chains yields often quite precise information about differences in the masses of their members. It is therefore fortunate that new information on $\alpha$ decay is still regularly reported, by laboratories in Finland, Germany, Japan and the USA.

We may remind you that for even-even nuclides, like the ${ }^{148}$ Dy and ${ }^{158}$ Dy mentioned above, $\alpha$-particle energies immediately determine their $\alpha$-decay energies, since the strongest feeding occurs to the daughter's ground-state. Also, no complications are known to occur here due to isomerism. The only trouble that could occur is, that the nuclide involved is misassigned; but this is expected to occur only rarely.

Unfortunately, this is not true for other nuclides. There is a large number of cases, where no information is available about the levels, that are fed in the $\alpha$-decay of nuclides with an odd number of neutrons and/or protons. Rather long ago, we tried to get some information about the average energy of the levels, fed by the highest energy $\alpha$-particles, by comparing the values for a chain of successive ones with those of neighbouring eveneven chains. They did not appear to differ much. Thus, we provisionally accepted them as due to transitions between ground-states. But in order to take the uncertainty into account, a value of $50 \mathrm{keV}$ is (quadratically) added to the reported errors in the resulting $\alpha$-decay energies. In principle, a better solution would be to accept the final level as an independent variable and to assign to its excitation energy some value with, say, an error 50. But we doubt that the extra complication due to the hundreds of new variables would be worth the trouble.

In the case that $\alpha$-decay is observed for two isomers, it is rather unlikely that they 
preferentially feed the same level in the daughter nuclide. Often, the resulting problem can partially be solved by the fact that isomerism also occurs in the daughter. By measuring correlations, one can often decide which daughter-isomer is fed by which parent. And, as said, results for isomers decaying by proton emission can also be valuable in desentangling the complications due to the occurence of isomers.

The fact mentioned above, that mass specroscopic results now start to become available for several members of an $\alpha$-decay chain is a help in alleviating the above problem. Yet, it does not eliminate it as long as the errors in those mass measurements are rather larger than those in $\alpha$-ray energies.

\section{Masses at the highest mass numbers}

Since Ame95, a further new element, $Z=112$, was discovered at Gsi (21), with mass number 277, the highest yet. Only two cases were found. A very remarkable fact was, that the two atoms of the daughter, ${ }^{273} 110$, occured after delays that were a factor 1000 different. Also, the most delayed one had a very significantly $(1.3 \mathrm{MeV})$ lower $\alpha$-energy. This points to the influence of a semi-magic number of neutrons.

Other reports on $\alpha$-decays in this neigbourhood also give very wellcome new information.

Not important for the mass work, but interesting to notice is, that the International Union of Pure and Applied Chemistry now accepted a set of names for the elements 103-109 (22). No names have yet been proposed for the last three known elements.

In the high mass region too, long chains of $\alpha$-decays are known. Again, the excitation energies of levels fed by the observed $\alpha$-rays are often not known. A help is here, that the prominent decays are regularly those to states with the same Nilsson model quantumnumbers as the parent (favored $\alpha$-decays). Differences between the positions of such particle-levels are often known for isotones or isotopes; and they do not change drastically as a function of $\mathrm{N}$ or $\mathrm{Z}$. We made a study of them, which allows us to make decent estimates for corresponding excitation energies of states fed in favored $\alpha$-decays.

In principle, the same could be done in the mass number region 160-200. We have started to analyze them too. The situation here, though, does not yet look less promising.

\section{Masses from iso-multiplet mass equations}

Recently, several cases have been studied of very proton-rich nuclides that $\beta$-decay to levels emitting protons, of which the energies are measured. Among them may be protons coming from the isobaric analogue of the mother nuclide. This may then permit to derive a decent mass value for that isobaric analogue. Authors then calculate the mass difference with the mother isotope from the (quadratic) Isobaric Multiplet Mass Equation (IMME). Before we discuss the consequences, the following is of interest.

A regular occurrence is, that an atomic mass value derived from a certain experiment disagrees rather severely with the value we would expect from extrapolation of masses of neighbouring nuclides (systematics). The difference may be outside the experimental error; but it may occur too that the reported error is compatible with the difference but (of course, then) quite large. In both cases, we had the habit to report both values. Earlier, the systematic value was given in the main table; but in our last table, AmE95, 
we there give the experimental value but with a flag warning that a probably better value derived from systematics is given in a separate table.

We consider to use a similar method in cases where mass values for proton-rich nuclides can be derived from consideration of isobaric analogue states. Mass values may be known for three or more isobaric analogues, so that its mass value can be derived from the IMME. In some cases, an experimental value for that nucleus may be available too, but with a far larger error. Examples: ${ }^{28} \mathrm{~S}$ and ${ }^{40} \mathrm{Ti}$, where the experimental errors are $160 \mathrm{eV}$ but the ones in the ImME derived values about $14 \mathrm{keV}$. We would wellcome reactions to the proposal, to give then in the main table the experimental value, but with a flag; and the one influenced by IMME in the separate table.

But consider now the cases mentioned above, where the mass of an isobaric analogue is known from proton decay. The mass of the "mirror" of the mother isotope is always known. If no mass value is known for one more analogue, one can not apply ImME. But then one can use the observation, that the constants in IMME are somewhat regular functions of the mass number (23). Thus, using interpolated values, one can derive an estimate for the mass difference of the mother nuclide and its analogue.

The resulting mass of the mother may be compared with the one following from the kind of systematics mentioned above. And we have cases where they do not quite agree. Here again, we might mention one in the main table, with a flag; the other in the supplementary table. We would, in this case, value opinions, which of the two should appear in the main table

For completeness, it may be mentioned that we made an elaborate study of the applicability of Imмe, be it restricted to analogues of ground-states. As a result, we advocate to increase the errors for proton-rich nuclides derived from IMME alone by certain amounts. Also, earlier considerations did not take into account the difference between proton- and neutron-pairing energies, which one of the present authors noticed to have a not negligible influence on the constants in the IMME. Last but not least: in the second situation mentioned above, we think that care should be taken to select the constants used in deriving the mass of the mother in a way to agree with the mass of its "mirror". Authors of such work sometimes do not take this into account.

One might say, that possibly the IMME is not exact anyhow. In cases where dependable mass values for four or more isobaric analogues are known, though, it seems to work quite well. We therefore feel that, in the cases mentioned, the mirror nuclide should indeed be taken into account.

\section{Acknowledgment}

One of the authors (AHW) thanks the direction of NIKHEF, Amsterdam, for the permission to use the facilities of this Institute and help of some of its members even rather long after his retirement.

\section{References}

1. G. Audi and A. H. Wapstra, Nucl. Phys. A 595, 409-480 (1995).

2. G. Audi and A. H. Wapstra, Nucl. Phys. A 565, 1-397 (1993).

3. K. S. Kozier et al., Can. J. Physics 58, 1311-1316 (1980). 
4. I. Bergström et al., "An attempt to measure the proton mass .." presented at EnAm95, Arles, June 1995, Proceedings pp. 787-797 (1996).

5. G. L. Greene et al., Phys. Rev. Lett. 56, 819-822 (1986).

6. R. D. Deslattes et al., Annals of Physics 129, 378-434 (1980).

7. D. H. White et al.,, Nucl. Instrum. Meth. 66, 70-76 (1968).

8. S. A. Brindhaban et al., Nucl. Instrum. Meth. A 340, 436-441 (1994).

9. G.Audi et al., Nucl. Phys. A 624, 1-124 (1997).

10. Ames,F. et al Nucl. Phys. A to be published.

11. D.Beck et al., Nucl. Phys. A 626, 343c-352c (1997).

12. A. S. Jensen, P. G. Hansen and B.Jonson, Nucl. Phys. A 431, 393-418 (1984).

13. T. Radon et al., Phys. Rev. Lett. 78, 4701-4704 (1997).

14. T. Beha, Thesis München 1995.

15. T. F. Kerscher, Thesis München 1996.

16. C. N. Davids et al., Phys. Rev. Lett. 76, 592-595 (1996).

17. R. D. Page et al., Phys. Rev. C 53, 660-670 (1996).

18. C. N. Davids et al, Phys. Rev. C 55, 2255-2266 (1997).

19. R. J. Irvine at al., Phys. Rev. C 55, 1621-1624 (1997).

20. C. N. Davids et al., Phys. Rev. Lett. 80, 1849-1852 (1998).

21. S. Hofmann et al., Z. Phys. 354, 229-230 (1996).

22. Comm. Nomencl.Inorg. Chem., Pure and Applied Chem. 69, 2471-2473 (1997).

23. M. S. Antony, A.Pape et al, Atomic Data and Nuclear Data Tables 33, 447-478 (1985), 34, 279-299 (1986), 40, 5-56 (1988).

21. J. Batchelder et al., Phys. Rev. C 57, 1042-1046 (1998). 